He described 9 days of breathlessness, chest tightness, vomiting and abdominal pain. His chest was clear and heart sounds normal with a soft murmur. He appeared very anxious. There were two positive findings of significant concern; a 3 centimetre palpable liver and persistent hypertension of more than 160/120. A chest radiograph showed a large heart and fluid in the horizontal fissure. More focused history taking revealed the shortness of breath was worse on exertion and on lying down, and he had to prop himself up at night to sleep.

The history and examination was consistent with heart failure. An ECG showed right ventricular hypertrophy and widespread T-wave inversion. An echocardiogram showed a structurally normal heart, mild mitral and aortic regurgitation, severely impaired left ventricular function (fractional shortening 13\%) and bilateral pleural effusions. A provisional diagnosis of viral myocarditis was made. However, in addition to persistent hypertension, his urea was 30 and creatinine 523.

He was admitted to a Paediatric Intensive Care Unit where acute management included oxygen, fluid restriction, furosemide and a milrinone infusion. In view of the deranged renal profile he was discussed with the renal team. Renal ultrasound revealed small, scarred kidneys and calyceal dilatation. The diagnosis was end stage renal failure due to undiagnosed grade 4 vesico-ureteric reflux, hypertension secondary to chronic kidney disease, and dilated cardiomyopathy secondary to hypertension.

It is rare for a teenager to present in heart failure, having never been unwell before. Originally we thought this was a primary cardiac problem, but the learning point here is that despite antenatal screening and good health care, congenital renal disease can remain asymptomatic, and that end stage renal failure can present with heart failure.

\section{G40(P) IS THERE ROLE FOR ECULIZUMAB IN THE MANAGEMENT OF C3 GLOMERULOPATHY?}

${ }^{1} \mathrm{D}$ Stewart, ${ }^{1} \mathrm{D}$ Hughes, ${ }^{2} \mathrm{~L}$ Riach, ${ }^{2} \mathrm{C}$ Oxley, ${ }^{1} \mathrm{D}$ Athavale. ${ }^{1}$ Renal Unit, Royal Hospital for Children, Glasgow, UK; '2Paediatric Department, Royal Aberdeen Children's Hospital, Aberdeen, UK

\subsection{6/archdischild-2018-rcpch.38}

Introduction The new classification, and increasing recognition, of dense deposit disease (DDD) as a C3 glomerulopathy and an alternative complement pathway disorder suggests that eculizumab, an anti-C5 monoclonal antibody, may be beneficial in treatment. However, the use of eculizumab as an off-label and expensive treatment in paediatrics is limited with variable clinical response in literature.

Method We present the outcomes of 2 paediatric patients in Scotland who have received eculizumab for the management of DDD.

Results Case 1: A 10-year-old male presented with steroidresistant proteinuria and biopsy confirmed DDD. He was managed symptomatically with an angiotensin converting inhibitor and diuretics. Four years later, due to worsening symptoms (oedema, lethargy, poor growth), renal function deterioration, and elevated terminal complement complex (TCC) levels, eculizumab was commenced. Repeat biopsy demonstrated chronic irreversible damage but 25\% glomerular architecture preservation. Symptomatic improvement was seen along with a reduction in proteinuria and renal function stabilisation. After 24 months, eculizumab was discontinued with progressive renal function deterioration seen in the final months of therapy. Six months later, due to worsening of symptoms and increasing proteinuria, eculizumab was recommenced with symptomatic improvement noted.

Case 2: A 5-year-old girl presented with steroid-resistant haematuria and proteinuria and biopsy confirmed DDD. Sixteen months following diagnosis, she rapidly progressed towards end-stage renal disease. Haemodialysis was commenced following no benefit from immunosuppression (mycophenolate mofetil or plasmapheresis). TCC level was elevated and eculizumab treatment was commenced 18 months after diagnosis. Renal biopsy prior to treatment showed marked pathological changes in all visible glomeruli. However, due to lack of clinical response, eculizumab was discontinued after 2 months. The patient remaining dialysis dependent.

Conclusions Our experience with eculizumab has demonstrated variable results. Only one patient exhibited a positive clinical response. Eculizumab was administered to both patients at varying points in their clinical journey with differing progressive biopsy features. Our experience adds to the limited evidence in literature, suggesting that a role for eculizumab in DDD may be guided by clinical and pathological features. However, further trials of its use in paediatric DDD are clearly needed.

\section{British Inherited Metabolic Diseases Group and British Society of Paediatric Gastroenterology, Hepatology and Nutrition}

\section{G41 A JOINT COLLABORATIVE REGIONAL NETWORK APPROACH TO OVERCOME DIFFICULTIES IN DIAGNOSIS OF PAEDIATRIC COELIAC DISEASE}

${ }^{1}$ A Hakizimana, ${ }^{2} \mathrm{~N}$ Heather, ${ }^{3} \mathrm{~J}$ Pridgeon, ${ }^{4} \mathrm{~N}$ Ward, ${ }^{2} \mathrm{NA}$ Afzal. ${ }^{1}$ Faculty of Medicine, University of Southampton, Southampton, UK; ${ }^{2}$ Department of Paediatrics, Southampton Children's Hospital, Southampton, UK; ${ }^{3}$ Department of Paediatrics, Queen Alexandra Hospital, Portsmouth, UK; ${ }^{4}$ Department of Paediatrics, Hampshire Hospitals NHS Foundation Trust, Basingstoke, UK

\subsection{6/archdischild-2018-rcpch.39}

Aims Diagnosis of paediatric coeliac disease ( $\mathrm{pCD}$ ) continues to be a challenge despite the new NICE, BSPGHAN and ESPGHAN guidance. To understand a 'true abnormal serologic result' and the need for a duodenal biopsy, we aimed for a quality improving exercise in a regional network setting.

Methods A two staged quality approach was adopted:

- An online regional survey was conducted to investigate the currently used serologic screening tests for diagnosis of pCD in the region. Regional paediatricians and pathology laboratories were contacted by phone and email followed by an online survey conducted via the Network Website (AH, NA).

- A summary presentation of the findings, were presented at the annual regional network meeting followed by a rigorous discussion with active participation of immunologists, paediatric consultants and dieticians from the region.

Results 9/13 hospitals in the regional network participated in the survey.

For coeliac screening IgA Anti-tTG is used by all centres (IgA EMA in $7 / 9$ centres (77.8\%). 5/9 (55.6\%) centres routinely check IgA levels in all cases. $5 / 9$ centres $(55.6 \%)$ combined 